\title{
Effectiveness of an Outreach Model of Care for Rheumatology Specialty Clinics to an On- Reserve First Nations Community.
}

\author{
Sujay Nagaraj
}

Margaret Kargard

Clinical Services Lead, Siksika Health Services, Siksika Alberta.

Brenda Hemmelgarn

Department of Medicine and Department of Community Health Sciences Cumming School of Medicine, University of Calgary.

Marvin J. Fritzler

Department of Medicine, Cumming School of Medicine, University of Calgary.

Tyler White

Chief Executive Officer, Siksika Health Services, Siksika Alberta.

Cheryl Barnabe

Department of Medicine and Department of Community Health Sciences Cumming School of Medicine, University of Calgary

Follow the International Journal of Indigenous Health at:

https://jps.library.utoronto.ca/index.php/ijih/index

Recommended Citation:

Nagaraj, S., Kargard, M., Hemmelgarn, B., Fritzler, M. J., White, T., \& Barnabe, C. (2018). Effectiveness of an Outreach Model of Care for Rheumatology Specialty Clinics to an On-Reserve First Nations Community. IJIH, 13(1), 157 - 167. https;//doi.org/10.18357/ijih.v13i1.30315 


\title{
Effectiveness of an Outreach Model of Care for Rheumatology Specialty Clinics to an On-Reserve First Nations Community.
}

\begin{abstract}
A model of care consisting of rheumatology specialty services embedded in the primary care system on a First Nations reserve was instituted to reduce barriers to care and improve inflammatory arthritis outcomes for patients. We assessed the effectiveness of this model of care on disease activity measures and patient-reported outcomes over 7 years. Patients were enrolled in a longitudinal cohort at the Siksika Nation in Alberta. Clinical characteristics, treatment recommendations and disease activity measures were systematically recorded over follow-up. Mixed-model regression was performed to determine rates of change for continuous measures. 59 participants ( $78 \%$ female; $\mathrm{M}=47$ years, $S D=13$ ), predominantly with rheumatoid arthritis (RA; $n=36)$, were followed for an average of 29 months $(S D=$ 23). Swollen and tender joint counts decreased significantly (change per month: $-0.20,95 \%$ CI -0.29 to 0.10 , and $-0.20,95 \% \mathrm{CI}-0.34$ to -0.06 , respectively) but pain, physician global and function scores did not significantly improve (all $p>0.05$ ). Patient global evaluation scores worsened over time (change per month $0.08,95 \%$ CI 0.029 to $0.131, p=0.002$ ). Inflammatory markers improved at a slower rate in patients with incident compared to incident disease. Disease-modifying agents were escalated for moderate or high disease activity at $64 \%$ of RA visits, with justifications for not escalating or application of local treatment approaches in all but one instance. Despite improvement in swollen and tender joint counts and adherence to current treatment paradigms, patient-reported outcomes did not significantly improve during follow-up. Further innovation is required to meet relevant outcomes.
\end{abstract}

\section{Keywords}

Inflammatory arthritis, First Nations, Outcomes

\section{Introduction}

First Nations Canadians experience high prevalence rates of inflammatory arthritis (IA) that include rheumatoid arthritis (RA), systemic lupus erythematosus (SLE), and spondyloarthritis (SpA), and with more severe consequences compared to non-First Nations populations. RA affects two to three times as many First Nations people in Manitoba (Barnabe, Elias, Bartlett, Roos, \& Peschken, 2008) and Alberta (Barnabe et al., 2017), respectively, compared to non-Indigenous populations. SpA also occurs at 1.5 to 3 times the rate of non-Indigenous populations (Barnabe et al., 2017). Increased disease severity has been reflected through higher disease activity scores and a predilection to involvement of large joints, resulting in worse physical function compared to Caucasians (Peschken et al., 2010). Utilization of rheumatology specialty care is reduced among First Nations populations (Barnabe et al., 2017), potentially explained by geographic and economic barriers to health services, but also related to poor care experiences in the health system, and competing priorities of other health conditions and roles and responsibilities to family and community (Thurston et al., 2014). Along with cultural competency 
education for healthcare providers, the development and implementation of innovative models of care were proposed as solutions to improve arthritis care outcomes (Thurston et al., 2014).

The Arthritis Alliance of Canada, in collaboration with the Canadian Rheumatology Association's Optimal Care Committee, has championed the development and evaluation of models of care for IA to ensure high quality and evidence informed care is provided to Canadians (Ahluwalia, Frank, Mosher, \& Zummer, 2014; Barber et al., 2016). Here, we describe patient outcomes of a model of care for arthritis patients instituted at the Siksika Nation in Treaty 7 Territory, a First Nations reserve in southern Alberta with 6,000 residents and with a primary health clinic located 100 kilometers from the nearest tertiary care centre in Calgary, Alberta.

\section{Model of Care}

\section{Methods}

The Siksika Health \& Wellness Clinic is an established primary health centre providing a range of services, including family physician and nurse practitioner visits, laboratory and diagnostic imaging, and is co-located with dentistry, optometry, community health, mental health, pharmacy and home care services. A tripartite agreement exists between the First Nations and Inuit Health Branch, Alberta Region, and Siksika Nation to ensure autonomous delivery of health services. The Siksika Nation was the first community in Alberta to institute an alternative payment plan (salary-based model) for family physicians, and has successfully established outreach services in nephrology (Ward et al., 2013), pediatrics, and dermatology. Rheumatology specialty services were added in 2010, accepting patients through both self-referral (advertised through a variety of community recruitment strategies such as advertisements through local media, recruitment at the local health fair or a community research assistant) and provider referral (i.e., family physician or nurse practitioner) mechanisms at weekly clinics held over 14 months in 2011-2012. This schedule was thereafter modified to a monthly clinic. In the interval between scheduled rheumatologist clinics, patients with inflammatory arthritis conditions are assessed by the primary healthcare providers in the community, who would directly consult with the rheumatologist as needed. Support from local nurses providing clinical care through a home care program was provided by the Nation.

\section{Participants}

From all patients assessed in the model of care during the time frame of the study $(n=271)$, consenting patients with IA conditions were enrolled into a longitudinal cohort to capture clinical diagnosis and disease characteristics, treatment recommendations and disease activity measures. We included participants enrolled between June 2011 and March 2016, with follow-up data available to March 2017. 


\section{Measures}

At the initial visit, clinical diagnosis, demographics (age, sex), comorbid medical conditions, smoking status, family history of rheumatic disease, serology (anti-nuclear antibodies, ANA, at a serum dilution of 1/160), rheumatoid factor (RF), anti-cyclic citrullinated peptide antibodies (ACPA), disease characteristics for all patients with IA, and treatment history for patients with prevalent IA were recorded. Disease activity measures (Tender Joint Count (TJC), Swollen Joint Count (SJC), Physician Global Score (MD Global), erythrocyte sedimentation rate (ESR), C-reactive protein (CRP), and patientreported outcomes (Patient Global Evaluation Score, Pt Global), Health Assessment Questionnaire (HAQ), Fatigue Visual Analog Scale (VAS) Pain VAS, and Sleep VAS were systematically recorded at baseline and over the course of follow-up. Additionally, the DAS28 score based on 28 joint counts and using the ESR (or CRP) were calculated for patients diagnosed with RA. For treatment strategy, each individual at each visit was classified as: having received a steroid injection (either intramuscular for bridging, or intra-articular/intra-bursal for disease management); recommended to maintain current treatment (i.e. no treatment changes required); taper therapy; initiate a new non-biologic diseasemodifying anti-rheumatic drug (DMARD); initiate a new biologic DMARD; or continue/use an oral steroid.

\section{Outcomes}

Change in disease-activity measures (TJC, SJC, CRP, ESR, MD Global) and patient-reported outcomes (HAQ, Fatigue VAS, Pain VAS, Sleep VAS, Pt Global) was evaluated for all patients. Changes in disease-activity measures that were specific to RA (DAS28 ESR/CRP) were additionally evaluated, as was a comparison between incident and prevalent cases. We determined if therapy was escalated for RA participants in moderate and high disease activity states (DAS28ESR >3.2, Fransen \& van Riel, 2006; or DAS28CRP > 2.9, Kuriya et al., 2017) if DAS28ESR not available according to a treat to target strategy, with escalation defined as either the addition of a new non-biologic or biologic DMARD, and also including the use of steroid (oral and parenteral) in the definition.

\section{Statistical Analysis}

Baseline disease activity, patient-reported outcomes, disease duration (for prevalent cases), demographics, and prognostic factors for participants were descriptively summarized. Changes in disease activity measures and patient-reported outcomes were estimated with multilevel mixed-effects linear regression models, adjusting for baseline HAQ score, age, gender, smoking status, as well as baseline SJC28 and TJC28. Comparisons in baseline characteristics between prevalent and incident cases and longitudinal changes in disease activity for both groups were done using t-tests and chi-square tests. All analyses were performed using STATA (version 14).

\section{Relationship}

Ethics. In collaboration with Siksika Health \& Wellness and the Siksika Nation, a memorandum of understanding and a research agreement for all parties involved was drafted and implemented. This 
study was conducted with heed to the Ownership, Control, Access and Possession (OCAP®) principles for research with First Nations communities. OCAP ${ }^{\circledR}$ is a registered trademark of the First Nations Information Governance Centre (FNIGC)((FNIGC), 2011). Individual consent was obtained following ethics approval obtained through the Conjoint Health Research Ethics Board at the University of Calgary.

\section{Cohort}

\section{Results}

In total, 59 patients with IA conditions, from 76 IA patients assessed by the rheumatologist, provided consent to participate in the longitudinal study. They were $78 \%$ female, with a mean age of 47 years $(S D=13)$ (Table 1). Clinical diagnoses included 36 with RA; 8 with SLE and related connective tissue diseases; 7 with Psoriatic arthritis (PsA); 3 with Juvenile Idiopathic Arthritis (JIA); 2 with SpA, 2 with crystal arthritis; and 1 person with undetermined IA. Thirty-one patients (53\%) had incident, and 28 $(47 \%)$ had prevalent disease. The mean disease duration in prevalent cases was $16(S D=13)$ years. At baseline, $63 \%$ of participants had at least one comorbidity, with the mean number of comorbidities for this group being $2.2(S D=1.1)$. Smoking was frequent with $79 \%$ of participants being current or past smokers.

\section{Table 1.}

Characteristics of Participants at Study Inception. Results are reported as mean (SD) or \% as appropriate, unless indicated.

\begin{tabular}{|c|c|c|c|}
\hline & $\begin{array}{c}\text { Total IA cohort }(n= \\
59)\end{array}$ & $\begin{array}{c}\text { Prevalent Cases }(n= \\
28)\end{array}$ & $\begin{array}{c}\text { Incident Cases }(n= \\
31)\end{array}$ \\
\hline Age & $47.2(12.6)$ & $47.2(13.4)$ & $47.2(12.1)$ \\
\hline Female & $78 \%$ & $86 \%$ & $71 \%$ \\
\hline Smoker (Current or past) & $78 \%$ & $76 \%$ & $81 \%$ \\
\hline Proportion with comorbidity * & $63 \%$ & $54 \%$ & $71 \%$ \\
\hline Number of comorbidities $* *$ & $2.2(1.1)$ & $2.2(1.1)$ & $2.3(1.1)$ \\
\hline Tender Joint Count (/28) & $8.7(7.7)$ & $6.4(6.8)$ & $10.8(7.9)$ \\
\hline Tender Joint Count (/68) & $15.6(11.3)$ & $11.3(10.3)$ & $19.3(10.9)$ \\
\hline Swollen Joint Count (/28) & $5.5(5.7)$ & $4.9(6.1)$ & $5.9(5.5)$ \\
\hline Swollen Joint Count (/66) & $9.7(8.5)$ & $8.0(8.9)$ & $11.1(8.0)$ \\
\hline DAS28 ESR (if RA) & $4.4(1.3)$ & $4.2(1.5)$ & $4.5(1.0)$ \\
\hline DAS28 CRP (if RA) & $4.5(1.6)$ & $4.4(1.7)$ & $4.5(1.5)$ \\
\hline Health Assessment Questionnaire & $1.35(0.71)$ & $1.51(0.65)$ & $1.22(0.74)$ \\
\hline Pain (0-10 Visual Analog Scale) & $6.2(2.5)$ & $5.9(2.6)$ & $6.4(2.5)$ \\
\hline Patient Global Evaluation Score (0-10 Visual Analog S.) & $5.0(2.6)$ & $4.8(2.7)$ & $5.1(2.5)$ \\
\hline Physician Global Evaluation Score (0-10 Visual Analog S.) & $3.5(2.8)$ & $2.9(3.1)$ & $4.4(2.1)$ \\
\hline Erythrocyte Sedimentation Rate (mm/hr) & $21.2(22.1)$ & $21.5(23.4)$ & $21.0(21.5)$ \\
\hline C-Reactive Protein $(\mathrm{mg} / \mathrm{dL})$ & $0.8(1.4)$ & $0.7(1.0)$ & $0.8 \quad(1.7)$ \\
\hline \multicolumn{4}{|c|}{$\begin{array}{l}\text { Note: Results are reported as mean (SD) or \% as appropriate, unless indicated. } \\
\text { * Comorbidities recorded include ischemic heart disease, transient ischemic attack, hypertension, hepatitis, kidney disease, diabetes, } \\
\text { osteoarthritis, and cancer } \\
\text { ** Calculated if at least one comorbidity reported } \\
\text { Legend: IA Inflammatory Arthritis; DAS28 ESR Disease Activity Score based on } 28 \text { joints including ESR; DAS28 CRP Disease } \\
\text { Activity Score based on } 28 \text { joints including CRP }\end{array}$} \\
\hline
\end{tabular}


Of the 36 participants with RA, $81 \%$ were seropositive at baseline. Additionally, $49 \%$ of participants diagnosed with RA or SLE were ANA positive at the time of assessment (41\% of RA; $86 \%$ of SLE patients). An analysis of ANA patterns and titres for patients with RA or SLE showed high frequencies of homogenous (60\%) and speckled (73\%) patterns with similar distributions of low (80, $160)$ and high $(>=320)$ titres (41\% and 59\%, respectively). Past medication use for prevalent cases included non-biologic DMARDs (including methotrexate, plaquenil, sulfasalazine, leflunomide, azathioprine and mycophenolate mofetil) in $32 \%$ of patients $(n=9 / 28)$ and biologic DMARDs in 5 patients $(18 \%)$. There were 4 patients $(7 \%)$ taking oral steroids at enrolment to the cohort.

\section{Disease Activity at Initial Assessment}

The mean baseline HAQ was $1.35(S D=0.71)$ and the mean TJC28 and SJC28 were $8.7(S D=$ 7.7) and $5.5(S D=5.7)$, respectively. Tender joint counts were higher but there were otherwise no differences in joint involvement, disease activity measures and patient-reported outcomes between incident and prevalent disease patients. Over 2/3's of RA patients $(72 \%)$ were in moderate or high DAS28 disease activity states at the initial visit.

Follow-up duration. A total of 374 visits were included in our analysis. Patients were followed for an average of $29(S D=23)$ months with an average of $6(S D=5)$ visits per participant and a mean of $6(S D=6)$ months in between consecutive visits. Eleven participants $(19 \%)$ were lost to follow-up. Disease activity and patient-reported outcomes over time. With adjustment for baseline HAQ, age, gender, smoking, baseline SJC28, and baseline TJC28, swollen and tender joint counts significantly improved during follow-up (SJC28 monthly rate of change $-0.20,95 \%$ CI -0.29 to -0.10 ; TJC28 monthly rate of change $-0.20,95 \% \mathrm{CI}-0.34$ to -0.01 ; both $p<0.01$ ). Although the swollen joint count based on 66 joints also demonstrated improvement (SJC66 monthly rate of change $-0.31,95 \%$ CI -0.46 to $-0.16, p<$ 0.001), TJC68 did not improve over time. Pain, ESR, CRP, MD Global, and HAQ scores, and DAS28ESR and DAS28CRP scores for RA patients, also did not significantly improve over time (Table 2). Patient global evaluation score worsened over time. When comparing disease activity over time between patients with incident and prevalent IA conditions, ESR and CRP were observed to improve at a slower rate for patients with incident disease (slope difference ESR 0.21, 95\%CI 0.01 to 0.41, p=0.04; slope difference CRP $0.02,95 \%$ CI 0.01 to $0.03, p<0.01$ ), whereas there were no differences in rate of change in the other disease activity measures between prevalent and incident cases (Table 2). 


\section{Table 2.}

Adjusted Monthly Rate of Change in Disease Activity Measures and Patient-Reported Outcomes.

\begin{tabular}{|c|c|c|}
\hline Outcome & Crude Slope (95\% CI) & Adjusted Slope * \\
\hline DAS28 ESR & $-0.45(-0.09$ to 0.01$) p=0.06$ & $-0.04(-0.09$ to 0.01$) p=0.15$ \\
\hline DAS28 CRP & $-0.03(-0.08$ to 0.03$) p=0.38$ & $-0.02(95 \% \mathrm{CI}-0.07$ to 0.04$) p=0.60$ \\
\hline Erythrocyte Sedimentation Rate & $-0.16(-0.46$ to 0.15$) p=0.31$ & $-0.28(95 \%$ CI -0.59 to 0.02$) p=0.070$ \\
\hline C-Reactive Protein & $-0.01(-0.03$ to 0.02$) p=0.68$ & $0.01(95 \% \mathrm{CI}-0.01$ to 0.03$) p=0.42$ \\
\hline Health Assessment Questionnaire & $0.01(-0.01$ to 0.01$) p=0.72$ & $-0.01(95 \%$ CI -0.01 to 0.01$) p=0.45$ \\
\hline Patient Global Evaluation Score & $0.08(0.03$ to 0.13$) p<0.01$ & $0.08(95 \%$ CI 0.03 to 0.13$) p=0.002$ \\
\hline Physician Global Evaluation Score & $-0.04(-0.09$ to 0.01$) p=0.15$ & $-0.05(95 \%$ CI -0.11 to 0.06$) p=0.078$ \\
\hline Pain & $0.013(-0.032$ to 0.057$) p=0.58$ & $0.01(95 \% \mathrm{CI}-0.05$ to 0.05$) p=0.97$ \\
\hline Tender Joint Count (/28) & $-0.19(-0.31$ to -0.07$) p<0.01$ & $-0.20(95 \% \mathrm{CI}-0.34$ to -0.06$) p<0.01$ \\
\hline Tender Joint Count (/68) & $-0.26(-0.47$ to -0.05$) p=0.01$ & $-0.22(95 \%$ CI -0.45 to 0.02$) p=0.07$ \\
\hline Swollen Joint Count (/28) & $-0.18(-0.27$ to -0.09$) p<0.01$ & $-0.20(95 \%$ CI -0.29 to -0.10$) p<0.01$ \\
\hline Swollen Joint Count (/66) & $-0.29(-0.43$ to -0.15$) p<0.01$ & $-0.31(95 \%$ CI -0.46 to -0.16$) p<0.01$ \\
\hline
\end{tabular}

Note: *Adjusted for baseline health assessment questionnaire score, age, gender, smoking status, baseline swollen joint count (28 joint count), and baseline tender joint count (28 joint count)

Treatment strategy. Treatment was declined at the time of recommendation at only $3 \%$ of visits ( $n=11 / 374$ ), with the current treatment plan maintained at $63 \%$ and therapy tapered at $4 \%$ of visits. New non-biologic DMARDS and biologic DMARDs were prescribed at $34 \%$ and $13 \%$ of visits respectively. Steroid injections (intra-muscular, intra-articular or soft tissue) were provided at $21 \%$ of visits. The treatment reported at follow-up visits $(n=315)$ included crystal arthritis treatment $(2 \%)$, nonbiologic DMARD monotherapy (21\%), non-biologic DMARD combination therapy (24\%), biologic DMARD monotherapy (6\%) or biologic DMARD in combination with non-biologic DMARD (6\%), oral steroids $(10 \%)$, or anti-inflammatories (18\%). At $14 \%$ of follow-up visits, the patients had not initiated recommended therapy, or had discontinued therapy at their discretion.

A total of 196 visits for RA occurred, of which 115 had data available to calculate disease activity state using either the DAS28ESR or DAS28CRP ( 71 of 81 visits without a calculable DAS28 score were related to missing inflammatory marker results). Remission was documented at $17 \%$, low disease activity at $19 \%$ and moderate or high disease activity at $64 \%$ of visits. Treatment was escalated with a biologic or non-biologic DMARD at $65 \%$ of visits where the patient was in moderate or high disease activity ( $n=48$ of 74 visits). Reasons for not escalating therapy with a biologic or non-biologic DMARD included initial visit status and baseline labs/investigations pending $(n=5)$, being too soon to assess response to a new therapy $(n=4)$, patient declining a therapy recommendation $(n=3)$, having a contraindication to therapy $(n=1)$, or physician-decision driven by the lack of swollen joints on 
assessment $(n=10)$ or opting to provide an intra-articular injection to a single swollen joint $(n=2)$. In a single case the current therapy was maintained despite active disease. There were no differences in escalation between incident and prevalent RA groups where RA patients were in moderate or high disease activity state $(p=0.7)$.

\section{Discussion}

We present effectiveness data from a model of care consisting of specialized rheumatology services embedded within the primary care home for an on-reserve First Nations community in Alberta. This model is designed to improve access to diagnosis and ensure sustained follow-up in keeping with established early and targeted treatment paradigms associated with improved inflammatory arthritis outcomes (Nam, 2016; Nell et al., 2004). Our model has had to balance the intake of patients with active disease that are prevalent cases, along with assessment and diagnosis of new patients who may or may not have inflammatory arthritis. Although there is no direct comparison possible for the level of access to rheumatology services existing before our model was established, we feel that the model is effective in ensuring access as evidenced through both the number of incident cases diagnosed, and a large volume of prevalent rheumatic disease patients returned to care, with a less than $20 \%$ loss to follow-up rate. This suggests an improvement to a barrier to care that patients may have previously experienced.

As evidenced by the disease activity at cohort inception there was a large unmet burden for both established and new IA cases as evidenced by a high percentage of individuals in moderate or high disease activity states (for RA cases), high swollen and tender joint counts as well as HAQ scores, and a paucity of those with prevalent arthritis being on therapy. Tender and swollen joints were observed in small, medium and large joints indicating the potential for a wide-spectrum of functional impairment. Patients showed significant improvements in these joint counts over the course of the study and treatment was escalated following the Treat to Target strategy with a relatively small proportion of patients electing to not pursue or discontinue therapy. It is interesting to note that there were no differences in rates of change in disease activity measures between prevalent and incident cases, though this is possibly due to the fact that those determined to have incident disease may have had a long symptom duration prior to presentation for diagnosis; unfortunately it is a limitation of the dataset that symptom duration at inception to the cohort was not collected.

Despite the suggested improvements in access and reductions in disease activity, we did observe an increase in patient global score and no significant improvements in other patient-reported outcomes over the duration of the study. The increase in patient global score may be attributable to arthritis progression, but also the various factors affecting health status beyond arthritis including but not limited to psychological distress, reduced mental health status (Euesden et al., 2017), and the impact of other medical comorbidities (Nikiphorou et al., 2016). Further, we have investigated these results through qualitative inquiry which will be presented in a future paper. In brief, the biomedical model, of which our program is an example, is not sufficient to meet the patient's emotional or spiritual needs, nor repair 
inequities that exist in the broader consideration of social determinants of health. Additionally, availability of patient support groups may be an integral part of treatment (Baker et al., 1996) but were not being offered in the community until recently. As such, it is suggested that it is necessary to address the social environments which play a role in affecting patient outcomes, and integrate these considerations in program development and advocacy work.

There are few rheumatology centres in Canada with clinical and research collaborations with First Nations populations, and this study was facilitated by the internal resources and strategic plan of a community recognized for innovations in health service delivery on the reserve. Our study contributes new knowledge to a prevalent and pressing issue in rheumatology care, and provides insights into how to enhance health service models to ensure that Indigenous communities in Canada receive adequate and accessible rheumatology care. Although efforts were taken to address limitations in this study, some still remain. Of particular importance is the proportion of participants who were lost to follow-up. These patients were not able to contribute information on whether they sought care elsewhere or made a decision to not pursue western medicine paradigms. These results may be mediated by many factors but we endeavour to seek ongoing feedback from the community and patients to mitigate further loss to follow-up. Further, there was only one rheumatologist who provided care for the entire duration of the study. A physician's experience in working with Indigenous communities and cross-cultural competency are not only assets but may be requirements to providing culturally-sensitive healthcare especially in a system with a legacy of racism towards Indigenous patients. It is also important to take note of the importance of allied health professionals and other community-health partnerships that must work in conjunction towards improving patient health. Additionally, our study only assessed one aspect of evaluation of a model of care: effectiveness at the patient-level. Ongoing evaluation of patient-level, provider-level and system-level outcomes are necessary (Dziedzic, French, Davis, Geelhoed, \& Porcheret, 2016) and are in progress (Barber, accepted). We address how this model of care can address the population of a particular Indigenous community but future studies should investigate the generalizability of such models of care in other communities.

\section{Conclusions}

Evaluation of the effectiveness of a model of care for patients with inflammatory arthritis implemented in a First Nations community highlighted areas for further improvement. Despite improvement in swollen and tender joint counts, patient-reported outcomes did not significantly improve during follow-up and gaps remain in meeting relevant outcomes.

\section{References}

(FNIGC), First Nations Information Governance Centre. (2011). The First Nations Principles of OCAP. Retrieved from www.FNIGC.ca/OCAP

Ahluwalia, V., Frank, C., Mosher, D., \& Zummer, M. (2014). A pan-Canadian Approach to Inflammatory Arthritis Models of Care. Retrieved from Toronto: www.arthritisalliance.ca 
Baker, P. R., Groh, J. D., Kraag, G. R., Tugwell, P., Wells, G. A., \& Boisvert, D. (1996). Impact of patient with patient interaction on perceived rheumatoid arthritis overall disease status.

Scandinavian Journal of Rheumatology, 25(4), 207-212. https://doi.org/10.3109/03009749609069989

Barber, C. E., Marshall, D. A., Mosher, D. P., Akhavan, P., Tucker, L., Houghton, K., . . A Arthritis Alliance of Canada Performance Measurement Development, Panel. (2016). Development of System-level Performance Measures for Evaluation of Models of Care for Inflammatory Arthritis in Canada. The Journal of rheumatology, 43(3), 530-540. https://doi.org/10.3899/jrheum.150839

Barnabe, C., Elias, B., Bartlett, J., Roos, L., \& Peschken, C. (2008). Arthritis in Aboriginal Manitobans: evidence for a high burden of disease. The Journal of rheumatology, 35(6), 1145-1150. Available from: http://www.jrheum.org/content/35/6/1145.long

Barnabe, C., Jones, C. A., Bernatsky, S., Peschken, C. A., Voaklander, D., Homik, J., . . Hemmelgarn, B. (2017). Inflammatory Arthritis Prevalence and Health Services Use in the First Nations and Non-First Nations Populations of Alberta, Canada. Arthritis care \& research, 69(4), 467-474. https://doi.org/10.1002/acr.22959

Dziedzic, K. S., French, S., Davis, A. M., Geelhoed, E., \& Porcheret, M. (2016). Implementation of musculoskeletal Models of Care in primary care settings: Theory, practice, evaluation and outcomes for musculoskeletal health in high-income economies. Best practice \& research. Clinical rheumatology, 30(3), 375-397.doi:10.1016/j.berh.2016.08.004

Euesden, J., Matcham, F., Hotopf, M., Steer, S., Cope, A. P., Lewis, C. M., \& Scott, I. C. (2017). The Relationship Between Mental Health, Disease Severity, and Genetic Risk for Depression in Early Rheumatoid Arthritis. Psychosom Med, 79(6), 638-645. https://doi.org10.1097/PSY.0000000000000462

Fransen, J., \& van Riel, P. L. (2006). DAS remission cut points. Clinical and experimental rheumatology, 24(6 Suppl 43), S-29-32. Available from: https://www.clinexprheumatol.org/article.asp?a=2935

Kuriya, B., Schieir, O., Lin, D., Xiong, J., Pope, J., Boire, G., . . Investigators, Catch. (2017). Thresholds for the 28-joint disease activity score (DAS28) using C-reactive protein are lower compared to DAS28 using erythrocyte sedimentation rate in early rheumatoid arthritis. Clinical and experimental rheumatology, 35(5), 799-803.

Nam, J. L. (2016). Rheumatoid arthritis management of early disease. Current opinion in rheumatology, 28(3), 267-274. https://doi.org/10.1097/BOR.0000000000000276

Nell, V. P., Machold, K. P., Eberl, G., Stamm, T. A., Uffmann, M., \& Smolen, J. S. (2004). Benefit of very early referral and very early therapy with disease-modifying anti-rheumatic drugs in patients with early rheumatoid arthritis. Rheumatology, 43(7), 906-914. https://doi.org/10.1093/rheumatology/keh199

Nikiphorou, E., Radner, H., Chatzidionysiou, K., Desthieux, C., Zabalan, C., van Eijk-Hustings, Y., . . . Gossec, L. (2016). Patient global assessment in measuring disease activity in rheumatoid arthritis: a review of the literature. Arthritis research \& therapy, 18(1), 251. https://doi.org/10.1186/s13075-016-1151-6

Peschken, C. A., Hitchon, C. A., Robinson, D. B., Smolik, I., Barnabe, C. R., Prematilake, S., \& ElGabalawy, H. S. (2010). Rheumatoid arthritis in a north american native population: longitudinal followup and comparison with a white population. The Journal of rheumatology, 37(8), 15891595. https://doi.org/10.3899/jrheum.091452 
Thurston, W. E., Coupal, S., Jones, C. A., Crowshoe, L. F., Marshall, D. A., Homik, J., \& Barnabe, C. (2014). Discordant indigenous and provider frames explain challenges in improving access to arthritis care: a qualitative study using constructivist grounded theory. Int J Equity Health, 13(1), 46. https://doi.org/10.1186/1475-9276-13-46

Ward, D. R., Novak, E., Scott-Douglas, N., Brar, S., White, M., \& Hemmelgarn, B. R. (2013). Assessment of the Siksika chronic disease nephropathy-prevention clinic. Canadian Family Physician, 59(1), e19-25. Available at:

https://pdfs.semanticscholar.org/6846/9486a0cfcb8ae965c950bc23f853c3485eed.pdf 